\title{
One Health Perspective on Infections in Nephrology: A Parallel Survey on Carbapenem- and Multidrug-resistant Klebsiella Pneumoniae and Pseudomonas Aeruginosa in High-risk Renal Patients and Farm Animals in Italy
}

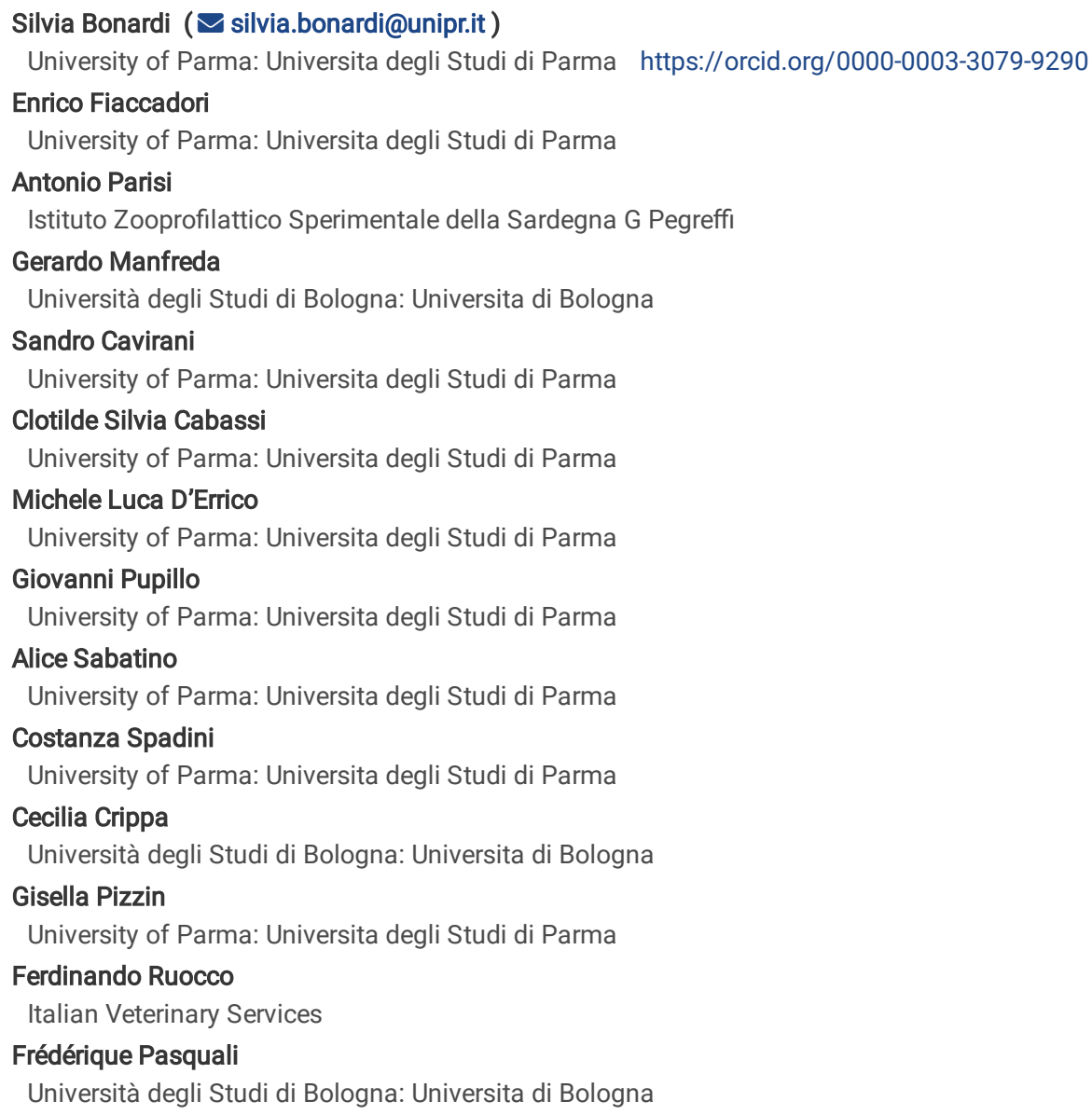

\section{Research}

Keywords: carbapenemases, Extended-Spectrum Beta-Lactamases, Klebsiella pneumoniae, Pseudomonas aeruginosa

Posted Date: May 5th, 2021

DOI: https://doi.org/10.21203/rs.3.rs-470540/v1

License: (9) (i) This work is licensed under a Creative Commons Attribution 4.0 International License. Read Full License 


\section{Abstract}

One Health is a multisectorial approach to achieve optimal health and well-being outcomes, recognizing the interconnections between people, animals, plants, and their environment. A relevant application is represented by antimicrobial resistance. Noteworthy, intensive farming has been frequently associated with abuse of antimicrobials and antimicrobial resistance is common among microorganisms from food-producing animals, whose transmission to humans cannot be excluded. In our survey, prevalence of carbapenem- and multidrug resistant $K$. pneumoniae and $P$. aeruginosa in renal patients and pigs in northern Italy was evaluated. During 2017-2018, 300 urine samples were collected from 187 high risk patients with kidney disease (mainly Acute Kidney Injury, 59/187, $31.5 \%$; or kidney transplant, 90/187, 48.1\%) at the Parma University Hospital. Additionally, 300 faecal samples were collected from slaughtered pigs. Carbapenemase-producing K. pneumoniae was detected in 4.8\% of patients (9/187). Specifically, ST554 b/a $a_{\mathrm{KPC}-3}$ (61.5\%), ST37 b/a $\mathrm{VIM}_{\mathrm{V}-1}$ (15.4\%), ST 258 bla $a_{\mathrm{KPC}-2}(15.4 \%)$ and ST4525 b/a $\mathrm{VIM}-1_{1}(7.7 \%)$ were found. To our knowledge, ST4525 is a novel ST-type and ST37 bla $a_{\mathrm{VIM}-1}$ was never reported before. ESBL genes ( $b / a_{\mathrm{SHV}}, b / a_{\mathrm{TEM}}, b / a_{\mathrm{Oxa}}$ and $\left.b / a_{\mathrm{CTX}}\right)$ were differently distributed among isolates, as well as several AMR genes. Carbapenem-resistant $K$. pneumoniae was not detected in pigs. Carbapenem-resistant $P$. aeruginosa was detected in $1.1 \%$ patients $(2 / 187)$ and in $1.3 \%$ pigs (4/300). Interestingly, $P$. aeruginosa ST938

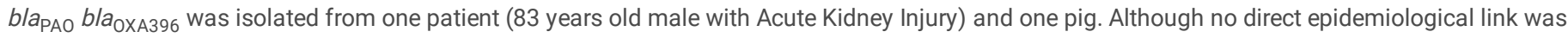
demonstrable, SNP calling suggested a close genetic relationship of the isolates (only 41 SNPs difference).

\section{Introduction}

One Health is a collaborative, multisectorial, and trans-disciplinary approach - working at local, regional, national, and global levels - to achieve optimal health and well-being outcomes, recognizing the interconnections between people, animals, plants and their shared environment; thus, the One Health concept is based on the definition of communication spaces among different environments and hosts, and antimicrobial resistance (AMR) represents an important field of this approach [1]. Resistance to carbapenems is of particular concern since they are the "last-line defence" to threat human infections by multiresistant Gram-negative bacilli. Carbapenem-resistance can be mediated either by porin deficiency combined with expression of AmpC- $\beta$-lactamases or ESBLs or by biosynthesis of carbapenemases [2]. Among carbapemases, class A KPC enzymes can hydrolyse all $\beta$-lactams in use. Class B enzymes belonging to the IMP, VIM and NDM families show the highest carbapenemase activity [3]. Class D carbapenem-hydrolyzing oxacillinases (OXA) have emerged in several bacterial species as responsible for carbapenem resistance [4]. The ESBLs (Extended-Spectrum $\beta$-Lactamases) hydrolyse most $\beta$-lactams but show a weak affinity for carbapenems. Families of ESBL enzymes include SHV, TEM-type-ESBLs and CTX-M [5].

Klebsiella pneumoniae and Pseudomonas aeruginosa infections are commonly healthcare associated. In Italy, $26.8 \%$ of $K$. pneumoniae and $15.8 \%$ of $P$. aeruginosa human invasive isolates were found to be carbapenem resistant (CR) in 2018 [6]. Hospital outbreaks have been predominantly attributed to $K$. pneumoniae ST258 bla KPC-3 $[7]$ but recently ST307 bla $a_{\mathrm{KPC}-3}$ and ST512 bla $\mathrm{KPC}-3_{3}$ have been emerging $[8,9]$ and a severe outbreak of NDM-1 producing $K$. pneumoniae has been reported [10].

Patients with kidney diseases are a population at high risk for multi drug resistance (MDR)-associated complications, as well a potential reservoir of antibioticresistant pathogens, owing to the coexistence of many factors increasing the risk of colonization and infection [11]. High prevalence of colonization/infection by MDR organisms has been documented in recent years in patients with kidney disease (both acute and chronic), who are disproportionally affected by antimicrobial resistance (AMR) as compared to the general population [11]. Along with cardiovascular disease, infection represents a leading cause of death among patients with chronic kidney disease, end-stage renal disease on dialysis and in those who have undergone kidney transplantation [12], as well as during and after an episode of Acute Kidney Injury (AKI) [13].

Carbapenems are not licenced for use in food-producing animals in the EU [14], but other $\beta$-lactams are commonly used, such as extended-spectrum cephalosporins, which could provide a selection pressure favouring the expression of carbapenem-resistance [15]. In the last decades, intensive farming has been frequently associated with use/abuse of antimicrobials and AMR is common among microorganisms from food-producing animals, whose transmission to humans cannot be excluded [16].

On these grounds, the present study was aimed at testing for CR K. pneumoniae and $P$. aeruginosa samples from both high-risk renal patients hospitalized in the Nephrology Unit at the Parma University Hospital and pigs at slaughter in the same area of the country (Parma, northern Italy).

The main objective of the study was focused on the One Health concept of AMR bacteria sharing between human and animal compartments.

The specific aims were focused on: $i$ ) the detection of CR bacteria in hospitalized patients and pigs; ii) the distribution of carbapenemases and other $\beta$ lactamases co-responsible for resistance to carbapenems in human and porcine strains; iii) the characterization of genetic clones of carbapenemaseproducing (CP) K. pneumoniae and P. aeruginosa; iv) the investigation on additional AMR genes in CR isolates.

\section{Materials And Methods}

\subsection{Sample collection}

\subsubsection{Patient sampling}

A total of 300 urine samples were collected from patients once every week in the Renal Intensive Care Unit (ICU) and the Renal Transplant Unit of the Parma University-Hospital. The samples were collected from indwelling bladder catheters in 187 patients (mean age 60 years, standard deviation 15) since 113 of them (60.4\%) were tested more than once because of prolonged hospitalization. The patients had been consecutively admitted between February 1 st, 2017 and May 31st, 2018 with a diagnosis mainly of AKI (59/187, 31.5\%) or kidney transplant (90/187, 48.1\%).

Page $2 / 13$ 
Written informed consent was obtained from the patients when possible or from their closest relatives when available. The Ethics Committee of the Area Vasta Emilia Nord (AVEN) approved the study (protocol n. 24497).

\subsection{Pig sampling}

From October 2016 to December 2017, 300 finishing pigs were randomly selected during 23 visits in a slaughterhouse of Parma province. The animals belonged to 300 batches and were reared in 95 farms. The caecal content of pigs was aseptically collected immediately after evisceration.

\subsection{Sample testing}

\subsubsection{Patient sample testing}

From each urine sample, $1 \mathrm{ml}$ was transferred into $5 \mathrm{ml}$ of Trypticase Soy Broth (TSB; Oxoid, Basingstoke, UK) added with a 10- $\mu$ g Meropenem disk (Oxoid). After overnight incubation at $35 \pm 2^{\circ} \mathrm{C}$ ), $100 \mu \mathrm{l}$ were streaked onto Mac Conkey agar (Oxoid) plates added with a 10- $\mu$ g Meropenem disk and $10 \mu \mathrm{l}$ were streaked onto Brilliance CRE Agar (Oxoid), which provides presumptive identification of $\mathrm{CR}$ microorganisms. Plates were incubated aerobically at $35 \pm 2{ }^{\circ} \mathrm{C}$ for 18-24 h. Suspect colonies were subcultured onto Trypticase Soy Agar plates (TSA; Oxoid) at $37 \pm 1^{\circ} \mathrm{C}$ for $18-22 \mathrm{~h}$ to obtain pure cultures for Gram-staining and further testing.

\subsubsection{Pig sample testing}

A $10 \mathrm{~g}$ aliquot of faecal material was suspended in $90 \mathrm{ml}$ of Buffered Peptone Water (BPW; Biolife Italiana, Italy) and incubated at $37 \pm 1^{\circ} \mathrm{C}$ for $18-22 \mathrm{~h}$. Thereafter, cultures plating and colonies selection were performed as described in 2.2.1.

\subsection{Kirby-Bauer Disk Diffusion Susceptibility test}

Human and porcine Gram-negative isolates were screened by the Kirby-Bauer Disk Diffusion Susceptibility test following EUCAST (2018) [17] and considering both clinical and screening breakpoints.

\subsection{Species identification}

Oxidase-negative isolates were tested by API® 20 E (bioMérieux, Marcy l'Etoile, France) following the manufacturer's instructions. Non-fermenting, oxidasepositive isolates were tested by the API 20 NE (bioMérieux) system.

\subsection{MIC test}

The CR isolates were analysed by the Minimal Inhibitory Concentration (MIC) test. Following EUCAST (2018) [17], K. pneumoniae and P. aeruginosa strains with meropenem MIC $>8 \mu \mathrm{g} / \mathrm{ml}$ (clinical breakpoint) and $K$. pneumoniae strains with meropenem MIC $>0.125 \mu \mathrm{g} / \mathrm{ml}$ (screening breakpoint) were selected for whole genome sequencing (WGS). E. coli ATCC 25922 was tested periodically as quality control microorganism.

\subsection{Whole genome sequencing}

Based on MIC, selected K. pneumoniae and $P$. aeruginosa isolates were tested by WGS. Whole-genomic DNA was extracted from $K$. pneumoniae and $P$. aeruginosa isolates using the MagAttract HMW DNA Kit (Qiagen, Hilden, Germany). The purified DNA concentration and the quality parameter ratio $260 / 280$ were measured by BioSpectrometer fluorescence (Eppendorf). Whole genomes were sequenced on Illumina MiSeq platform (Nextera library, paired-end reads). The INNUca v3.2 pipeline was used to quality check and de novo assemble reads into contigs. The pipeline includes SPAdes 3.11 as de novo assembler and provides Multi Locus Sequence Typing (MLST) profile.

SNP calling was performed on reads using snippy 4.0 pipeline with default setting (https://github.com/tseemann/snippy). An alignment of core SNPs was produced to infer a high-resolution phylogeny. A maximum likelihood (ML) tree was constructed using the iQTree program and was viewed using iTOL v4.2.3 software [18]. Klebsiella pneumoniae str. HS11286 (Ref Seq GCA_000240185.2) and Pseudomonas aeruginosa PA01 (Ref Seq NC_002516.2) were used as references for SNP calling on K. pneumoniae and $P$. aeruginosa newly sequenced reads, respectively.

Analysis of resistome of genomes was performed using ABRicate [19]. With this tool a BLAST search of genes included in the Resfinder database was performed on de novo assemblies [20, 21].

De novo assemblies were annotated by RAST version 2.0 [22]. Gbk files were used as input files for visualisation of the genetic environment of carbapenemase encoding genes by SnapGene [23]. Assembled sequences are available at NCBI [24] under BioProject PRJNA587603.

\section{Results}

\subsection{Klebsiella pneumoniae of human origin}

Seventeen K. pneumoniae strains were isolated from 12 patients (Table 1 ). Long-lasting infections in four patients were revealed by repeated detection of $K$. pneumoniae over time. 
Table 1

Carbapenem-resistant strains isolated from humans and pigs in the study period

\begin{tabular}{|c|c|c|c|c|}
\hline source & ID code & MIC values $(\mu \mathrm{g} / \mathrm{ml})$ & MLST & bla genes \\
\hline & \multicolumn{4}{|c|}{ K. pneumoniae } \\
\hline human & NEF 48 & $16 \pm 0$ & 37 & VIM-1, OXA-1, CTX-M-15, SHV-12 \\
\hline human & NEF 87 & $32 \pm 0$ & 258 & KPC-2, OXA-9, SHV-187 \\
\hline human & NEF 88 & $13.33 \pm 4.62$ & 258 & KPC-2, OXA-9, SHV-12 \\
\hline human & NEF 112 & $10.67 \pm 4.62$ & 37 & VIM-1, OXA-1, CTX-M-15 \\
\hline human & NEF 116 & $85.33 \pm 36.95$ & 554 & KPC-3, OXA-9, SHV-182 \\
\hline human & NEF 119 & $256 \pm 0$ & 554 & KPC-3, OXA-9, SHV-182 \\
\hline human & NEF 123 & $16 \pm 0$ & 4525 & VIM-1, OXA-1, CTX-M-15, SHV-12 \\
\hline human & NEF 130 & $>256$ & 554 & KPC-3, OXA-9, SHV-182 \\
\hline human & NEF 131 & $>256$ & 554 & KPC-3, OXA-9, SHV-182 \\
\hline human & NEF 145 & $>256$ & 554 & KPC-3, OXA-9, SHV-182 \\
\hline human & NEF 196/2 & $64 \pm 0$ & 554 & KPC-3, OXA-9, SHV-182 \\
\hline human & NEF 197 & $64 \pm 0$ & 554 & KPC-3, OXA-9, SHV-182 \\
\hline human & NEF 198 & $2 \pm 0$ & 277 & - \\
\hline human & NEF 199 & $1.67 \pm 0.58$ & 277 & SHV-27 \\
\hline human & NEF 261 & $0.027 \pm 0.009$ & 307 & SHV-28, CTX-M-15 \\
\hline human & NEF 278 & $0.15 \pm 0.1$ & 307 & OXA-1, SHV-106, CTX-M-15 \\
\hline \multirow[t]{2}{*}{ human } & NEF 297 & $53.33 \pm 18.48$ & 554 & KPC-3, OXA-9, SHV-182 \\
\hline & \multicolumn{4}{|c|}{ P. aeruginosa } \\
\hline human & NEF 23 & $21.33 \pm 9.24$ & 938 & OXA-396, PAO \\
\hline human & NEF 156 & $8 \pm 0$ & 395 & OXA-488, PAO \\
\hline pig & CRE 98 & $2 \pm 0$ & 274 & OXA-486, PAO \\
\hline pig & CRE 102 & $4.67 \pm 3.06$ & 938 & OXA-396, PAO \\
\hline pig & CRE 153 & $16 \pm 0$ & 782 & OXA-50, PAO \\
\hline pig & CRE 295 & $1.67 \pm 0.58$ & 885 & OXA-50, PAO \\
\hline
\end{tabular}

WGS identified carbapenemase genes in 13 (76.5\%) strains from 9 patients. Apparent prevalence of CP K. pneumoniae infected patients was $4.8 \%$ (9/187) $\mathrm{Cl}_{95}[2.6-8.9]$. Sequence identity ( $\geq 98 \%$ ) and coverage minimum sequence length ( $\left.>60 \%\right)$ were used as selection criteria for gene detections. The $13 \mathrm{CP} K$. pneumoniae isolates harboured bla $\mathrm{KPC}_{3}(61.5 \%)$, b/a $a_{\mathrm{VIM}-1}(23.1 \%)$ and $b / a_{\mathrm{KPC}-2}(15.4 \%)$, as well as other $\beta$-lactamase-producing (bla) genes (Tables 1 and 2$)$. CP K. pneumoniae belonged to ST554 (61.5\%), ST37 (15.4\%), ST258 (15.4\%) and ST4525 (7.7\%). The bla $a_{\mathrm{KPC}-2}$ and $b / a_{\mathrm{KPC}-3}$ determinants were linked to specific ST-types, namely ST258 and ST554, respectively. The bla $\mathrm{VIM}_{-1}$ gene was found in ST37 and ST4525 (Table 2). 
Table 2

Resistomes of CP K. pneumoniae strains of human origin

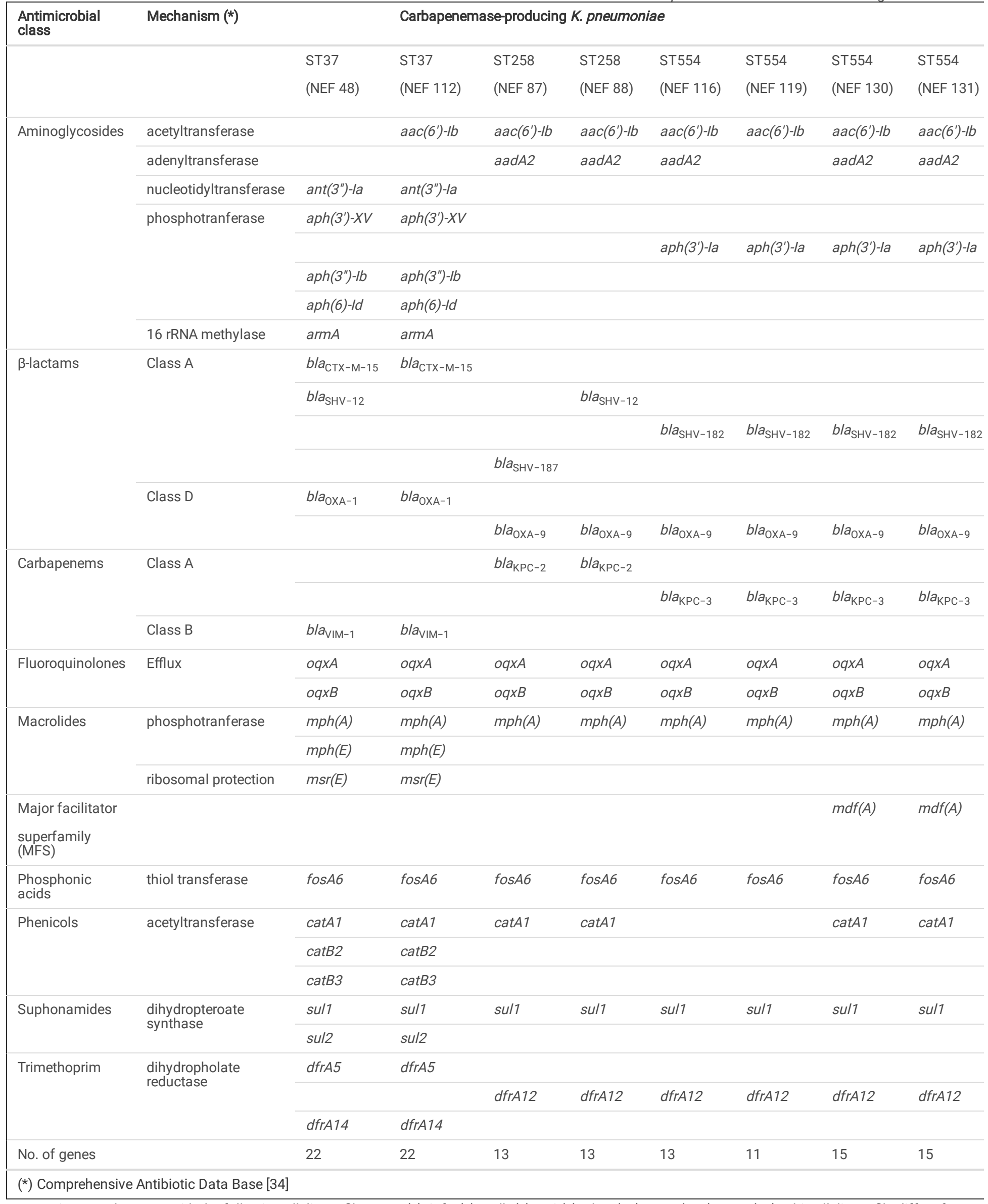

ST4525 is a novel ST-type with the following allelic profile: gapA (2); infB (9); mdh (2); pgi (1); phoE(13); rpoB (214); tonB (16). This allelic profile differs from ST37 by only one nucleotide within rpoB gene: T at position 100 of allele rpoB_1 (ST37) is substituted by a C in allele rpoB_214 (ST4525) (Fig. 1). 
In three K. pneumoniae isolates $(3 / 17 ; 17.6 \%)$, ESBL genes belonging to $b / a_{\mathrm{SHV}}, b / a_{\mathrm{TEM}}, b / a_{\mathrm{oxa}}$ and $b / a_{\mathrm{CTX}}$ families were found. These isolates were characterized by MIC values lower than the clinical breakpoint $(<2 \mu \mathrm{g} / \mathrm{ml})($ Table 3$)$.

Table 3

Resistomes of ESBL K. pneumoniae strains of human origin

\begin{tabular}{|c|c|c|c|c|}
\hline \multirow[t]{3}{*}{ Antimicrobial class } & \multirow[t]{3}{*}{ Mechanism * } & \multicolumn{3}{|c|}{ ESBL $K$. pneumoniae } \\
\hline & & ST277 & ST307 & ST307 \\
\hline & & (NEF 199) & (NEF 261) & (NEF 278) \\
\hline \multirow{6}{*}{$\begin{array}{l}\text { Aminoglycosides, } \\
\text { non-specific }\end{array}$} & \multirow[t]{2}{*}{ acetyltransferase } & & $\operatorname{aac}\left(3^{\prime}\right)-1 / a$ & $\operatorname{aac}\left(3^{\prime}\right)-1 / a$ \\
\hline & & & & $\operatorname{aac}\left(6^{\prime}\right)-1 b-c r_{-} 1$ \\
\hline & adenyltransferase & & aadA1_4 & \\
\hline & nucleotidyltransferase & & $\operatorname{ant}\left(3^{\prime \prime}\right)-1 a$ & \\
\hline & \multirow[t]{2}{*}{ phosphotranferase } & & $a p h(3 ")-1 b \_5$ & $a p h\left(3^{\prime \prime}\right)-1 b \_5$ \\
\hline & & & & $a p h(6)-I d$ \\
\hline \multirow[t]{6}{*}{$\beta$-lactams } & \multirow[t]{5}{*}{ Class A } & & & $b / a_{\mathrm{CTX}-\mathrm{M}-15}$ \\
\hline & & $b / a_{\mathrm{SHV}-27}$ & & \\
\hline & & & $b / a_{\mathrm{SHV}-106}$ & $b / a_{\mathrm{SHV}-106}$ \\
\hline & & & & $b / a_{\mathrm{TEM}-1 \mathrm{~B}}$ \\
\hline & & & $b / a_{\text {TEM-102 }}$ & \\
\hline & \multicolumn{3}{|l|}{ Class D } & $b l a_{O X A-1}$ \\
\hline \multirow[t]{3}{*}{ Fluoroquinolones } & \multirow[t]{3}{*}{ efflux } & oqxA & & oqxA \\
\hline & & $o q x B$ & & $o q x B$ \\
\hline & & & & qnrB1 \\
\hline Macrolides & \multirow[t]{2}{*}{ phosphotranferase } & & $m p h(E)$ & \\
\hline $\begin{array}{l}\text { Major facilitator } \\
\text { superfamily (MFS) }\end{array}$ & & $m d f(A)$ & $m d f(A)$ & $m d f(A)$ \\
\hline \multirow[t]{2}{*}{ Phosphonic acids } & \multirow[t]{2}{*}{ thiol transferase } & & fosA6 & \\
\hline & & & fosA7 & \\
\hline Suphonamides & dihydropteroate synthase & & & sul2 \\
\hline Tetracyclines & efflux & & & $\operatorname{tet}(A) \_6$ \\
\hline Trimethoprim & dihydropholate reductase & & & dfrA14_5 \\
\hline $\mathrm{N}^{\circ}$ of genes & & 4 & 10 & 15 \\
\hline
\end{tabular}

ST258 bla $a_{\mathrm{KPC}-2}$ isolates carried other bla genes, namely bla ${ }_{\mathrm{OXA}-9}, b / a_{\mathrm{SHV}-12}$, and b/a $\mathrm{SHV}_{-187}$. Their resistome consisted of 13 genes conferring resistance to nine antimicrobial classes. ST554 b/a $a_{\mathrm{KPC}-3}$ isolates carried bla $a_{\mathrm{OXA}-9}$ and b/a $a_{\mathrm{SHV}-182}$, with resistomes consisting of 11-15 genes. One ST37 bla $a_{\mathrm{VIM}-1}$ strain carried $b / a_{\mathrm{OXA}-1}$ and $b / a_{\mathrm{CTX}-\mathrm{M}-15}$, whereas the other ST37 bla $\mathrm{VIM}-1_{1}$ additioanlly carried bla $a_{\mathrm{SHV}-12}$. Their resistome consisted of 22 AMR genes. (Table 2 )

The novel ST4525 bla $a_{\mathrm{VIM}-1}$ (NEF123) was characterized by a resistome of 17 genes, which included $b / a_{\mathrm{CTX}-\mathrm{M}-15}, b / a_{\mathrm{SHV}-12}$, bla $a_{\mathrm{OXA}-1}$ and genes for resistance to aminoglycosides, fluoroquinolones, macrolides, fosfomycin, chloramphenicol, sulphonamides, and trimethoprim (Table 2).

Among the ESBL ST307 isolates, one strain harboured $b / a_{\mathrm{SHV}-106}$ and $b / a_{\mathrm{TEM}-102}$, and the other carried $b / a_{\mathrm{SHV}-106}, b / a_{\mathrm{TEM}-1 \mathrm{~B}}, b / a_{\mathrm{CTX}-\mathrm{M}-15}$ and $b / a_{\mathrm{OXA}-1}$. One

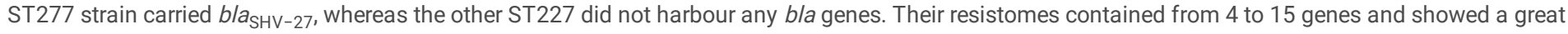
variety (Table 3 ).

\subsection{Pseudomonas aeruginosa of human and porcine origin}

Two CR P. aeruginosa strains were isolated from patients. Apparent prevalence was 1.1\% (2/187) (95\% Cl: 0.3\%-3.8\%) (Table 1). The NEF23 strain belonged to ST938 and its resistome consisted of five genes, including bla $a_{\text {OXA-396 }}$ and bla $a_{\text {PAO }}$ while the NEF156 strain belonged to ST395 and its resistome consisted of six genes, including $b / a_{\mathrm{OXA}-488}$ and bla $a_{\mathrm{PAO}}$ (Table 4). 
Table 4

Resistomes of $P$. aeruginosa of human and porcine origin

\begin{tabular}{|c|c|c|c|c|c|c|c|}
\hline \multirow[t]{3}{*}{ Antimicrobial class } & \multirow[t]{3}{*}{ Mechanism * } & \multicolumn{6}{|c|}{$P$. aeruginosa } \\
\hline & & ST-938 & ST-938 & ST-274 & ST-782 & ST-885 & ST-395 \\
\hline & & (NEF 23) & (CRE 102) & (CRE 98) & (CRE 153) & (CRE 295) & (NEF 156) \\
\hline Aminoglycosides, non-specific & phosphotransferase & $a p h\left(3^{\prime}\right)-I l b$ & $a p h\left(3^{\prime}\right)-I I b$ & $\mathrm{aph}\left(3^{\prime}\right)-11 \mathrm{~b}$ & $a p h\left(3^{\prime}\right)-I I b$ & $a p h\left(3^{\prime}\right)-I I b$ & $a p h\left(3^{\prime}\right)-11 b$ \\
\hline \multirow[t]{5}{*}{$\beta$-lactams } & \multirow[t]{4}{*}{ Class D } & & & & $b / a_{\mathrm{OXA}-50}$ & $b / a_{0 \times A-50}$ & \\
\hline & & $b / a_{\mathrm{OXA}-396}$ & $b / a_{0 \times A-396}$ & & & & \\
\hline & & & & \multicolumn{3}{|l|}{ bla OXA-486 } & \\
\hline & & & & & & & $b / a_{0 X A-488}$ \\
\hline & PAO genes & $b / a_{\mathrm{PAO}}$ & $b / a_{\mathrm{PAO}}$ & $b / a_{\mathrm{PAO}}$ & $b l a_{\mathrm{PAO}}$ & $b / a_{\mathrm{PAO}}$ & $b / a_{\mathrm{PAO}}$ \\
\hline Fluoroquinolones (Ciprofloxacin) & phosphorylase & & & crpP & crpP & crpP & crpP \\
\hline Phosphonic acids & thiol transferase & fosA4 & fosA4 & fosA4 & fosA4 & fosA4 & fosA4 \\
\hline Phenicols & acetyltransferase & catB7 & catB7 & catB7 & catB7 & catB7 & catB7 \\
\hline $\mathrm{N}^{\circ}$ of genes & & 5 & 5 & 6 & 6 & 6 & 6 \\
\hline
\end{tabular}

In pigs, four CR P. aeruginosa strains were detected. Specifically, one ST938 isolate (CRE102) was identical to the NEF23 from a 83-old male patient hospitalized one month after pig slaughter for an episode of AKI due to sepsis, on previous chronic kidney disease. Moreover, based on SNP calling, the two genomes differed by only 41 SNPs suggesting the close genetic relationship among the porcine and the human isolates (Fig. 3, Table S3). No direct epidemiological link was found.

The remaining $P$. aeruginosa isolates of porcine origin belonged to ST274, ST782 and ST885 and all of them harboured the bla $a_{\mathrm{PAO}} \mathrm{gene}$. In addition, $b / a_{\mathrm{OXA}-50}$ (ST782 and ST885) and bla OXA-486 (ST274) were identified together with other AMR genes (Tables 1 and 4).

\subsection{Single Nucleotide Polymorphisms (SNPs) calling}

Results on de novo assembly statistics are reported in table S1. SNP calling confirmed results of MLST typing with a higher level of discriminatory power (Figs. 2 and 3). Regarding K. pneumoniae, within the same ST types pairwise SNP differences were 41 (ST37), 51 (ST277), 55 (ST258), ranging from 29 and 89 (ST554) and 1071 (ST307). Isolate NEF123 (ST4525) showed 292 and 296 SNPs differences to NEF112 and NEF48 both belonging to ST37, demonstrating the close genetic relationship of the two ST-types (Fig. 2, table S2). Not surprisingly, between genomes of different ST-types the genetic distance increased with pairwise SNPs differences ranging from 905 to 32805.

Regarding Pseudomonas, CRE102 and NEF23 (ST938) of porcine and human origin respectively showed pairwise SNPs distances of 41 SNPs confirming their close genetic relationship (Fig. 3, table S3). Not surprisingly, comparing genomes of different ST-types (ST274, ST782, ST885, ST395) the genetic distance increased with pairwise SNPs differences ranging from 17359 to 20981.

\subsection{Localisation of carbapenemase-encoding genes}

Within the meropenem-resistant $K$. pneumoniae isolates, the carbapenemase-encoding genes $b / a_{\mathrm{KPC}-2}, b / a_{\mathrm{KPC}-3}$ or $b / a_{\mathrm{VIM}-1}$ were detected. To evaluate their mobilisation potential, their genetic environments were investigated. $b / a_{\mathrm{KPC}-2}$ and $b / a_{\mathrm{KPC}-3}$ were included in a complete Tn 4401 (Figs. 4 and 5 ). Tn 4401 was previously described as a transposon belonging to the Tn3 family ad involved in bla $a_{\mathrm{KPC}}$ mobilisation [25].

bla $\mathrm{VIM}-1_{1}$ was located close to other AMR determinant genes (Fig. 6). Although the contig was too short to confirm the localisation of the gene in a class 1 integron, the sequence showed $99 \%$ identity in comparison to $K$. pneumoniae genomes (CP032178.1, MF344563.1) in which the sequence was included in a class 1 integron within a transposon (data not shown). Moreover, this gene was already detected within class 1 integrons as part of a Tn402 transposon [26].

Regarding $P$. aeruginosa, bla $\mathrm{OXA}_{\mathrm{A}}$ and bla $\mathrm{PAO}_{\mathrm{O}}$ showed genetic environments which did not show any specific sequence of mobilizable genetic elements (MGEs) confirming previous data (Figs. 7 and 8) [27].

\section{Discussion}

\subsection{Klebsiella pneumoniae in humans}

In our study, K. pneumoniae ST258 bla KPC-2, which is one of the most common in Italian hospital settings [7], was identified in two patients only (22.2\%) tested on the same day. Instead, K. pneumoniae ST554 bla KPC-3 has been the only clone isolated from the two renal wards for 10 months (July $2017-M a y ~ 2018)$, suggesting a long-lasting outbreak involving six patients. MDR to amynoglicosides, $\beta$-lactams, macrolides, phenicols, sulphonamides, fluoroquinolones and fosfomycin was associated to carbapenem resistance. 
To our knowledge, this is the first report of K. pneumoniae ST37 producing VIM-1 in Italy. The two isolates were detected from the same patient (72 years old male with AKI) tested twice during a three-month period (April-July 2017. The isolates carried the bla $\mathrm{VIM}_{1}$ gene within a class 1 integron, along with additional ESBL-encoding genes $\left(b / a_{\mathrm{CTX}-\mathrm{M}-15}, b / a_{\mathrm{SHV}-12}, b / a_{\mathrm{OXA}-1}\right)$ and several AMR determinants. High levels of AMR were identified also in the new sequence type ST4525 bla $a_{\mathrm{VIM}-1}$ (NEF123), including ESBL genes (b/a $\mathrm{CTX}_{\mathrm{M}-15}, b_{\mathrm{SHV}-12}$, bla $a_{\mathrm{OXA}-1)}$ and genes for resistance to aminoglycosides, fluoroquinolones, macrolides, fosfomycin, chloramphenicol, sulphonamides, and trimethoprim.

\subsection{K. pneumoniae in pigs}

K. pneumoniae has been associated with sporadic cases of disease in pigs, including pneumonia, mastitis and septicaemia [28]. In our study, CR $K$. pneumoniae was never isolated from swine, not only because carbapenems are banned in veterinary treatments, but also for the high biosecurity measures commonly applied in pig farms, which exclude access of pigs to the natural environment or anthropic sources of AMR bacteria [29].

\subsection{Pseudomonas aeruginosa in humans}

Although P. aeruginosa $\beta$-lactams resistance in usually mediated by the chromosomally encoded AmpC type $\beta$-lactamase induced by contacts with sub-MIC levels of $\beta$-lactams, the microorganism can acquire resistance through chromosomal mutations as well as acquisition of antimicrobial resistance genes horizontally transferred [30].

The two CR ST938 and ST395 strains did not harbour carbapenemase-encoding genes, but bla $a_{\text {OXA }}$ and blapAO genes, confirming that decreased susceptibility to meropenem could be conferred by oxacillinases [31].

\subsection{Pseudomonas aeruginosa in pigs}

In most of cases, $P$. aeruginosa has not a primary role in pig infections [32]. Interestingly, two porcine strains harboured $\beta$-lactamases genes, such as bla $a_{0 X A-50}$ which is an intrinsic oxacillinase able to confer decreased susceptibility to ampicillin, ticarcillin and meropenem [31].

\subsection{Shared $P$. aeruginosa ST938 between humans and pigs and implications for safety}

This is the first report of an ESBL-MDR $P$. aeruginosa strain isolated both from humans and pigs in Italy, whose resistance to carbapenems is due to $\beta$ lactamases genes other than carbapenemases. P. aeruginosa ST938 carrying bla $a_{\text {OXA-396 }}$ and bla $a_{\text {PAO }}$ was isolated from a pig slaughtered in February 2017 and from an 83-old male patient hospitalized one month later for an episode of AKI due to sepsis, on previous chronic kidney disease. Even if no epidemiological links between these findings were found, the occurrence of the same CR strain in humans and pigs reflects the One Health concept of bacterial sharing between humans and food animals.

Since other isolates of MDR $P$. aeruginosa harbouring ESBL genes were found in pigs, the potential risk of pig meat contamination and subsequent human exposure to MDR opportunistic pathogens could not be excluded. Especially raw pork and products thereof (e.g. sausages, salami) could represent a risk for humans.

\section{Conclusions}

In recent years, the concept of One Health has been reformulated underlying the role of geographically close ecosystems in the occurrence of traits which have an impact on human, animal, plant, and environmental health, such as AMR [33].

In our study based on the One Heath perspective of bacterial exchange between human and animal compartments, K. pneumoniae strains harbouring b/a $a_{\mathrm{KPC}-2}$, $b / a_{\mathrm{KPC}-3}$ and b/a $a_{\mathrm{VIM}-1}$ were isolated from patients only. This finding is consistent with the European ban of carbapenem use in food-producing animals. Nevertheless, despite treatments bans, environmental overlapping could cause microbial and MGEs exchange between humans and animals, causing wide distribution of AMR bacteria in different settings. On the contrary, $P$. aeruginosa was found both in human and porcine entities, but the isolates did not carry carbapenemase genes and their carbapenem-resistance was associated to bla $a_{0 X A-50}$ family and blapAO genes. In addition, MDR microorganisms were mostly detected in K. pneumoniae strains from hospitalized patients, probably because of the selection pressure following antimicrobial treatments. The surprising similarity in AMR of $P$. aeruginosa strains detected from pigs and patients could be due to improper use in pig husbandry of the same antimicrobial agents used in the hospital setting. However, the circulation of bacterial strains between the animal and human compartments cannot be excluded

\section{Declarations}

Funding:

the study was funded by Cariparma Foundation Grants No 2016.0108 and No 2018.0176

\section{Conflicts of interest:}

The authors declare no conflicts of interest.

\section{Ethics approval:}


The study adhered to the Helsinki Declaration for Ethical Treatment of Human Subjects, with local ethics committee approval (The Ethics Committee of the Area Vasta Emilia Nord, AVEN n. 24497). The study respects international ethics standards for anonymity and data protection.

\section{Consent to participate:}

Written informed consent was obtained from the patients when possible or from their closest relatives when available.

\section{Availability of data:}

Silvia Bonardi, Enrico Fiaccadori and Frederique Pasquali had full access to all of the data in the study and take responsibility for the integrity of the data and the accuracy of the data analysis.

\section{Code availability:}

not applicable

\section{Authors' contributions:}

S.B., E.F., F.P.: study design, data analysis; paper writing A.P: WGS analyses; C.S.C., C.S., M.L.D.E.: MIC testing; G.M., S.C: paper supervision; G.P., G.P.: microbiological testing; C.C.: data analyses; A.S.; F.R.: samples collection.

\section{References}

1. Baquero F, Coque TM, Martinez J-L, Aracil-Gisbert S, Lanza VF. Gene transmission in the One Health microbiosphere and the channels of antimicrobial resistance. Frontiers Microbiol https://. 2019. doi:10.3389/fmicb.2019.02892.

2. Jeon JH, Lee JH, Lee JJ, Park KS, Karim AM, Lee CR, et al. Structural basis for carbapenem-hydrolyzing mechanisms of carbapenemases conferring antibiotic resistance. Int J Mol Sci. 2015;16:9654-92. https://doi:10.3390/ijms16059654.

3. Nordmann P, Naas T, Poirel L. Global spread of carbapenemase producing Enterobacteriaceae. Emerg Infect Dis. 2011;17:1791-8. doi:10.3201/eid1710.110655.

4. Naas T, Oueslati S, Bonnin RA, Dabos ML, Zavala A, Dortet L, et al. Beta-lactamase database (BLDB)-structure and function. J Enzyme Inhib Med Chem. 2017;32:917-9. doi:10.1080/14756366.2017.1344235.

5. Thomson KS. Extended-spectrum- $\beta$-lactamase, AmpC, and carbapenemase issues. J Clin Microbiol. 2010;48:1019-25. https://doi:10.1128/JCM.0021910.

6. European Centre for Disease Prevention and Control (ECDC). (2019) Surveillance Atlas of Infectious Diseases https://atlas.ecdc.europa.eu/public/index.aspx Accessed 14 May 2020.

7. Villa L, Feudi C, Fortini D, Brisse S, Passet V, Bonura C, et al. (2017). Diversity, virulence, and antimicrobial resistance of the KPC- producing Klebsiella pneumoniae ST307 clone. Microb Genome 3: e 000110. https://doi:10.1099/mgen.0.000110.

8. Calia C, Pazzani C, Oliva M, Scrascia M, Lovreglio P, Capolongo C, et al. Carbapenemases-producing Klebsiella pneumoniae in hospitals of two regions of Southern Italy. APMIS. 2017;125:491-8. https://doi:10.1111/apm.12666.

9. Rimoldi SG, Gentile B, Pagani C, Di Gregorio A, Anselmo A, Palozzi AM, et al. Whole genome sequencing for the molecular characterization of carbapenem-resistant Klebsiella pneumoniae strains isolated at the Italian ASST Fatebenefratelli Sacco Hospital, 2012-2014. BMC Infect Dis. 2017;17:1-11. doi:10.1186/s12879-017-2760-7.

10. Tavoschi L, Forni S, Porretta A, et al. (2020) Prolonged outbreak of New Delhi metallo-beta-lactamase-producing carbapenem-resistant Enterobacterales (NDM-CRE), Tuscany, Italy, 2018 to 2019. Euro Surveill 25: 2000085. https://doi: 10.2807/1560-7917.ES.2020.25.6.2000085.

11. Wang TZ, Kodiyanplakkal RPL, Calfee DP. Antimicrobial resistance in nephrology. Nat Rev Nephrol. 2019;15:463-81. doi:10.1038/s41581-019-0150-7.

12. Saran R, Robinson B, Abbott KC, Agodoa LYC, Bragg-Gresham J, Balkrishnan R, et al. (2019) US Renal Data System 2018 Annual Data Report: Epidemiology of Kidney Disease in the United States. Am J Kidney Dis 73: A7-8. doi:10.1053/j.ajkd.2019.01.001.

13. Silver SA, Harel Z, McArthur E, Nash DM, Acedillo R, Kitchlu A, et al. Causes of death after a hospitalization with AKI. J Am Soc Nephrol. 2018;29:1001-10. https://doi:10.1681/ASN.2017080882.

14. Roschanski N, Friese A, von Salviati- Claudius C, Hering J, Kaesbohrer A, Kreienbrock L, et al. Prevalence of carbapenemase producing Enterobacteriaceae isolated from German pig-fattening farms during the years 2011-2013. Vet Microbiol. 2017;200:124-9. https://doi:10.1016/j.vetmic.2015.11.030.

15. Mollenkopf DF, Stull JW, Mathys DA, Bowman AS, Feicht SM, Grooters SV, Daniels JB, Wittuma TE. Carbapenemase-Producing Enterobacteriaceae Recovered from the Environment of a Swine Farrow-to-Finish Operation in the United States. Antimicrob Agents Chemother. 2017;61:e01298-16.

16. Food and Agricolture Organization of the United Nations (FAO), Office International des Epizooties (OIE), and World Health Organization (WHO). (2019). Monitoring and evaluation of the global action plan on antimicrobial resistance: framework and recommended indicators. http: www.who.int Accessed January 2, 2020. 
17. European Society of Clinical Microbiology and Infectious Diseases (EUCAST). (2018). EUCAST breakpoints. Eur. Comm. Antimicrob. Susceptibility Testing.

http://www.eucast.org/fileadmin/src/media/PDFs/EUCAST_files/Breakpoint_tables/v_8.1_Breakpoint_Tables.pdf\%0Ahttp://www.eucast.org/fileadmin/srı

18. Interactive Tree of Life (iTOL). Klebsiella pneumoniae. (2021) https://itol.embl.de/ Accessed 1 March 1, 2021.

19. ABRicate. (2021) https://github.com/tseemann/abricate/ Accessed 11 March 2021.

20. Center for Genomic Epidemiology. (2021) K. pneumoniae. https://cge.cbs.dtu.dk/services/ResFinder/ Accessed 1 March 2021.

21. Center for Genomic Epidemiology. (2021) P. aeruginosa. https://cge.cbs.dtu.dk/services/ ResFinder/ Accessed 1 March 2021.

22. Overbeek R, Olson R, Pusch GD, Olsen GJ, Davis JJ, Disz T, Edwards RA, Gerdes S, Parrello B, Shukla M, Vonstein V, Wattam AR, Xia F, Stevens R. The SEED and the Rapid Annotation of microbial genomes using Subsystems Technology (RAST). Nucleic Acids Res. 2014;2:D206-14. doi. 10.1093/nar/gkt1226.

23. SnapGene. (2021) https://www.snapgene.com/ Accessed 1 March 2021.

24. National Center for Biotechnology Information. (2021) http: //www.ncbi.nlm.nih.gov Accessed 1 March 2021.

25. Cuzon G, Naas T, Nordmann P. Functional characterization of Tn4401, a Tn3-based transposon involved in bla KPC gene mobilization. Antimicrob Agents Chemother. 2011;55:5370-3. https://doi:10.1128/AAC.05202-11.

26. Tato M, Coque TM, Baquero F, Cantón R. Dispersal of carbapenemase blaVIM-1 gene associated with different Tn402 variants, mercury transposons, and conjugative plasmids in Enterobacteriaceae and Pseudomonas aeruginosa. Antimicrob Agents Chemother. 2010;54:320-7. https://doi:10.1128/AAC.00783-09.

27. Walther-Rasmussen J, Høiby N. OXA-type carbapenemases. J Antimicrob Chemother. 2006;57:373-83. htpps://doi:10.1093/jac/dki482.

28. Broes A, Taylor DJ, Martineau GP. Miscellaneus bacterial infections. In: Zimmerman JJ, Karriker LA, Ramirez A, Schwartz KJ, Stevenson GW, Zhang J, editors. Diseases of Swine. New York: Wiley; 2019. pp. 981-1001.

29. Martinez JL. Environmental pollution by antibiotics and by antibiotic resistance determinants. Environl Pollut. 2009;157:2893-902. doi. 10.1086/514909.

30. Botelho J, Grosso F, Peixe L. Antibiotic resistance in Pseudomonas aeruginosa - Mechanisms, epidemiology and evolution. Drug Resist Updat https://. 2009. doi:10.1016/j.drup.2019.07.002.

31. Girlich D, Naas T, Nordmann P. Biochemical characterization of the naturally occurring oxacillinase OXA-50 of Pseudomonas aeruginosa. Antimicrob Agents Chemother. 2004;48:2043-8. doi:10.1128/AAC.48.6.2043-2048.2004. https.

32. Leman AD, Straw BE, Mengeling WL, Sylvie D, Taylor DJ. Diseases of Swine. 7th ed.: lowa State University Press; 1992.

33. van Bruggen AHC, Goss EM, Havelaar A, van Diepeningen AD, Finckh MR, Morris JG Jr. One Health - Cycling of diverse microbial communities as a connecting force for soil, plant, animal, human and ecosystem health. Sci Total Environ. 2019;664:927-37. doi. 10.1016/j.scitotenv.2019.02.091.

34. Comprehensive Antibiotic Data Base. https:// card.mcmaster.ca Accessed 1 March 2021.

\section{Figures}




\begin{tabular}{|c|c|c|}
\hline rPOB1_NEF48 & ACCCACTACGGTCGCGTATGTCCGATCGAAACGCCTGAAGGTCCGAACATCGGTCTGATT & 60 \\
\hline rPOB1_NEF112 & ACCCACTACGGTCGCGTATGTCCGATCGAAACGCCTGAAGGTCCGAACATCGGTCTGATT & 60 \\
\hline \multirow[t]{2}{*}{ rPOB214_NEF123 } & ACCCACTACGGTCGCGTATGTCCGATCGAAACGCCTGAAGGTCCGAACATCGGTCTGATT & 60 \\
\hline & & \\
\hline rPOB1_NEF48 & AACTCCCTGTCCGTGTACGCGCAGACCAACGAATATGGCTTCCTTGAGACGCCGTATCGT & 120 \\
\hline RPOB1_NEF112 & AACTCCCTGTCCGTGTACGCGCAGACCAACGAATATGGCTTCCTTGAGACGCCGTATCGT & 120 \\
\hline \multirow[t]{2}{*}{ rPOB214_NEF123 } & AACTCCCTGTCCGTGTACGCGCAGACCAACGAATATGGCCTCCTTGAGACGCCGTATCGT & 120 \\
\hline & 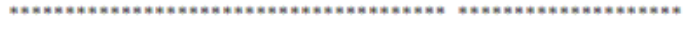 & \\
\hline rPOB1_NEF48 & AAAGTGACCAACGGTGTGGTTACTGACGAAATTCACTACCTGTCTGCTATCGAAGAAGGC & 180 \\
\hline RPOB1_NEF112 & AAAGTGACCAACGGTGTGGTTACTGACGAAATTCACTACCTGTCTGCTATCGAAGAAGGC & 180 \\
\hline \multirow[t]{2}{*}{ IPOB214_NEF123 } & AAAGTGACCAACGGTGTGGTTACTGACGAAATTCACTACCTGTCTGCTATCGAAGAAGGC & 180 \\
\hline & 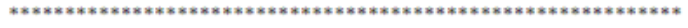 & \\
\hline rPOB1_NEF48 & AACTACGTTATCGCTCAGGCGAACTCCAACCTGGATGAAAACGGCCACTTCGTAGAAGAT & 240 \\
\hline RPOB1_NEF112 & AACTACGTTATCGCTCAGGCGAACTCCAACCTGGATGAAAACGGCCACTTCGTAGAAGAT & 240 \\
\hline \multirow[t]{2}{*}{ IPOB214_NEF123 } & AACTACGTTATCGCTCAGGCGAACTCCAACCTGGATGAAAACGGCCACTTCGTAGAAGAT & 240 \\
\hline & $* * * * * * * * * * * * * * * * * * * * * * * * * * * * * * * * * * * * * * * * * * * * * * * * * * * * * * * * * * * * * * *$ & \\
\hline rPOB1_NEF48 & CTGGTTACCTGCCGTAGCAAAGGCGAATCCAGCTTGTTCAGCCGCGACCAGGTTGACTAC & 300 \\
\hline RPOB1_NEF112 & CTGGTTACCTGCCGTAGCAAAGGCGAATCCAGCTTGTTCAGCCGCGACCAGGTTGACTAC & 300 \\
\hline \multirow[t]{2}{*}{ rPOB214_NEF123 } & CTGGTTACCTGCCGTAGCAAAGGCGAATCCAGCTTGTTCAGCCGCGACCAGGTTGACTAC & 300 \\
\hline & 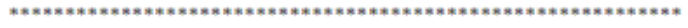 & \\
\hline rPOB1_NEF48 & ATGGACGTATCCACCCAGCAGGTGGTATCCGTCGGTGCGTCCCTGATCCCGTTCCTGGAA & 360 \\
\hline RPOB1_NEF112 & ATGGACGTATCCACCCAGCAGGTGGTATCCGTCGGTGCGTCCCTGATCCCGTTCCTGGAA & 360 \\
\hline \multirow[t]{2}{*}{ rPOB214_NEF123 } & ATGGACGTATCCACCCAGCAGGTGGTATCCGTCGGTGCGTCCCTGATCCCGTTCCTGGAA & 360 \\
\hline & 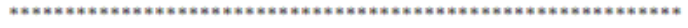 & \\
\hline rPOB1_NEF48 & CACGATGACGCCAACCGTGCATTGATGGGTGCGAACATGCAACGTCAGGCGGTTCCGACT & 420 \\
\hline RPOB1_NEF112 & CACGATGACGCCAACCGTGCATTGATGGGTGCGAACATGCAACGTCAGGCGGTTCCGACT & 420 \\
\hline \multirow[t]{2}{*}{ TPOB214_NEF123 } & CACGATGACGCCAACCGTGCATTGATGGGTGCGAACATGCAACGTCAGGCGGTTCCGACT & 420 \\
\hline & 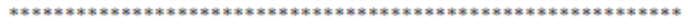 & \\
\hline rPOB1_NEF48 & CTGCGCGCTGATAAGCCGCTGGTTGGTACCGGTATGGAACGTGCTGTTGCCGTTGACTCC & 480 \\
\hline rPOB1_NEF112 & CTGCGCGCTGATAAGCCGCTGGTTGGTACCGGTATGGAACGTGCTGTTGCCGTTGACTCC & 480 \\
\hline \multirow[t]{2}{*}{ rPOB214_NEF123 } & CTGCGCGCTGATAAGCCGCTGGTTGGTACCGGTATGGAACGTGCTGTTGCCGTTGACTCC & 480 \\
\hline & & \\
\hline гров1 & GGTGTTACTGCCGTGGCTAAA & \\
\hline rPOB1_NEF112 & GGTGTTACTGCCGTGGCTAAA & \\
\hline rPOB214_NEF123 & GGTGTTACTGCCGTGGCTAAA & \\
\hline
\end{tabular}

\section{Figure 1}

alligment of rpoB gene of NEF 48 (ST37), NEF112 (ST37) and NEF123 (ST4525)

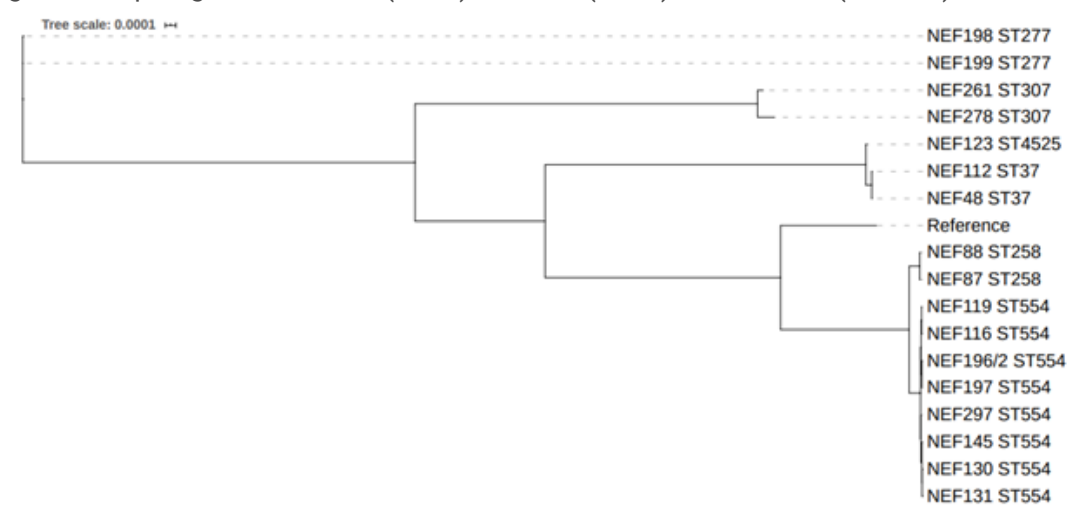

Figure 2

Core SNPs Maximum Likelihood tree showing genetic relationships among K. pneumoniae genomes included in this study and the reference Klebsiella pneumoniae str. HS11286 (Ref Seq GCA_000240185.2)

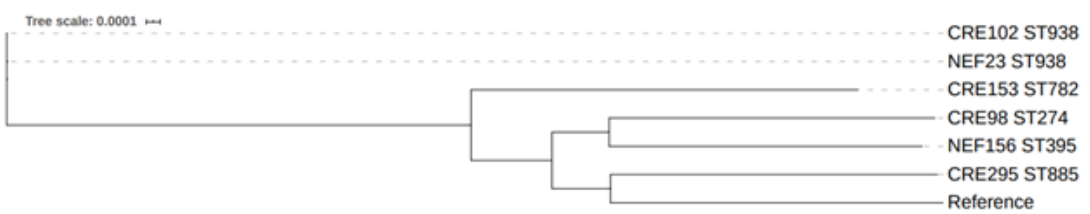

Figure 3

Core SNPs Maximum Likelihood tree showing genetic relationships among P. aeruginosa and P. fluorescens genomes included in this study and the reference P. aeruginosa PA01 (Ref Seq NC_002516.2) 


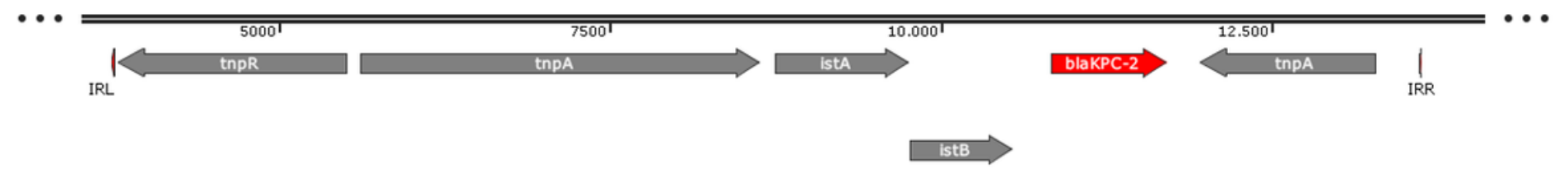

NEF87_NEF88_Tn4401_blaKPC-2

\section{Figure 4}

Genetic environment of blaKPC-2 gene in NEF 87 (ST258). The same genetic environment was observed in NEF 88 (ST258) genome

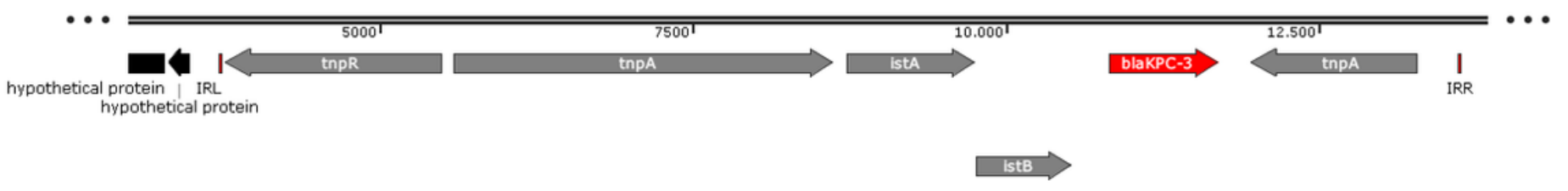

NEF297_NODE68_Tn4401_blaKPC-3

20.505 bp

\section{Figure 5}

Genetic environment of blaKPC-3 gene in NEF197 (ST554) genome. The same genetic environment was observed in NEF 116, NEF119, NEF 130, NEF 131, NEF 145, NEF 196/2, NEF 197 (ST554) genomes

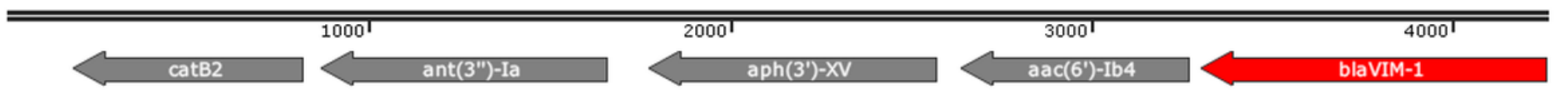

NEF48_NODE57_int1_blaVIM1

$4258 \mathrm{bp}$

\section{Figure 6}

Genetic environment of blaVIM-1 gene in NEF 48 (ST37) genome. The same genetic environment was observed in NEF 112 (ST37) and NEF 123 (ST4525) genomes. 


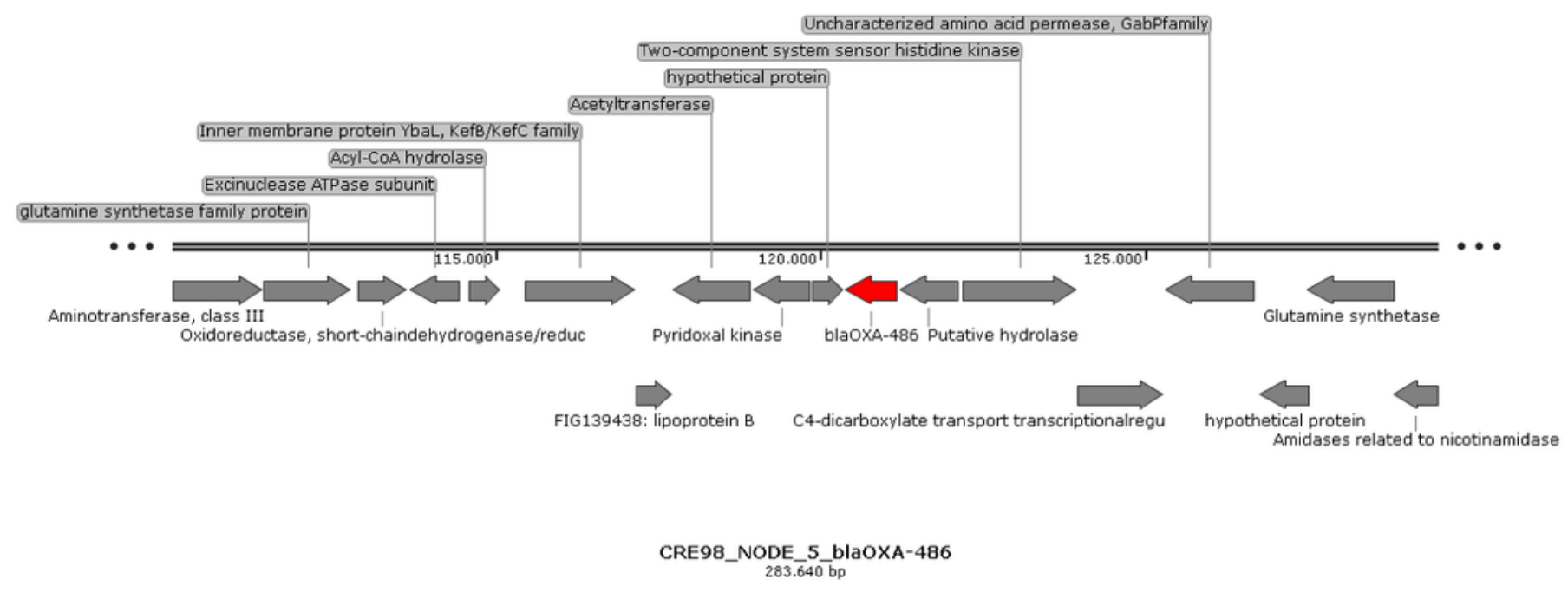

Figure 7

Genetic environment of blaOXA-486 gene in CRE 98 (ST274). The same genetic environment was observed for all detected genes belonging to the blaOXA-50 family in NEF 23 (ST938) , CRE 102 (ST938), CRE 153 (ST782), CRE 295 (ST885) and NEF 156 (ST395) genomes

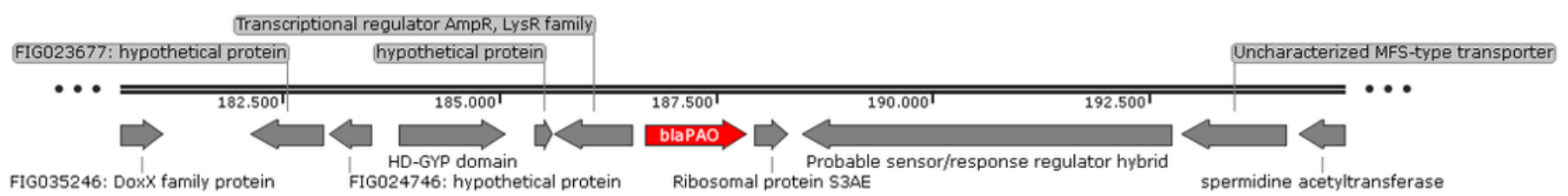

FIG005107: hypothetical protein

\section{CRE98_NODE_7_blaPAO \\ $215.299 \mathrm{bp}$}

\section{Figure 8}

Genetic environment of blaPAO gene in CRE 98 (ST274) genome. The same genetic environment was observed in NEF 23 (ST938) , CRE 102 (ST938), CRE 153 (ST782), CRE 295 (ST885) and NEF 156 (ST395) genomes

\section{Supplementary Files}

This is a list of supplementary files associated with this preprint. Click to download.

- Supplementarytables.docx 